\title{
O treinamento muscular inspiratório incrementa o equilíbrio postural em pacientes submetidos à reabilitação cardíaca: um estudo piloto
}

\author{
The inspiratory muscle training improves postural balance in patients \\ undergoing cardiac rehabilitation: a pilot study
}

\author{
Juliani Lemes Sbeghen, Tamires Daros dos Santos, Isabella Martins de Albuquerque
}

Como citar este artigo: SBECHEN, JULIANI L: SANTOS, TAMIRES D.; ALBUQUERQUE, ISABELLA M.: $\bigcirc$ treinamento muscular inspiratório incrementa o equilibrio postural em pacientes submetidos à reabilitação cardíaca: um estudo piloto. Revista Saúde (Sta. Maria). 2021; 47 (1).

Autor correspondente: Nome: Juliani Lemes Sbeghen

Telefone: (55) 99193025 E-mail: julianisbeghen87@ gmail.com

Formação Profissional: Graduanda do curso de Fisioterapia da Universidade Federal de Santa Maria (UFSM), Santa Maria, RS, Brasil, Bolsista do Programa Institucional de Bolsas de Iniciação Científica (PIBIC) do Conselho Nacional de Desenvolvimento Científico e Tecnológico (CNPq).

Filiação Institucional: Universidade Federal de Santa Maria

Endereço para correspondência:

Rua: Onofre Gonçalves Ferrão n: 270

Bairro: Tomazetti

Cidade: Santa Maria

Estado: RS

CEP: 97065-270

Data de Submissão: 28/09/2020

Data de aceite: 13/05/202

Conflito de Interes se: Não há conflito de interesse

(cc) $\mathrm{Br}-\mathrm{NC}-\mathrm{ND}$

\section{RESUMO}

Objetivo: Avaliar os efeitos do treinamento muscular inspiratório (TMI) de moderada a alta intensidade associado à reabilitação cardíaca $(\mathrm{RC})$ no equilíbrio postural, força muscular inspiratória e periférica, medo de queda e mobilidade funcional de pacientes inseridos na Fase II da RC. Métodos: Trata-se de um estudo de delineamento do tipo quaseexperimental. Previamente as avaliações iniciais, 12 indivíduos foram randomizados em grupo treinamento muscular inspiratório (GTMI, $n=7$ ), com carga de treinamento de moderada a alta intensidade ou em grupo treinamento muscular inspiratório Sham (GTMIS, $n=5$ ) com carga mínima mantida constante. Os seguintes desfechos foram avaliados: equilíbrio postural (Berg Balance Scale-BBS e Balance Evaluation Systems Test-BESTest); força muscular inspiratória (pressão inspiratória máxima-PImáx); força muscular periférica (teste de uma repetição máxima-1RM); confiança auto-percebida (Activities-specific Balance Confidence-ABC); mobilidade funcional (Timed Up and Go-TUG e teste de velocidade da marcha de 6 metros). 0 protocolo de treinamento consistiu em TMI, exercício aeróbico e resistido durante 12 semanas, com duas sessões semanais. Resultados: Os achados deste estudo piloto demonstraram que após intervenção, na comparação intra grupo, o GTMI apresentou incremento no escore do BESTest (pré 94,7 pontos vs pós 101 pontos, $p=<0,001$ ), na PImáx expressos em valores absolutos (pré $81,43 \mathrm{cmH} 20$ vs pós 105,86 cmH2O, $p=0,006$ ) e na porcentagem atingida do predito (pré $89,37 \%$ vs pós $115,10 \%, p=0,002$ ), assim como, a redução no tempo do TUG (pré 8,04 s vs pós 6,54 s, $p=0,009$ ). Na comparação entre os grupos, houve aumento no escore do BESTest $(p=0,049)$ e na PImáx expressos em valores absolutos $(p=0,041)$ e na porcentagem atingida do predito $(p=0,017)$ à favor do GTMI. Porém, sem diferença significativa para os demais desfechos avaliados. Conclusão: Os resultados deste estudo piloto sugerem que o TMI de moderada a alta intensidade associado à RC propiciou incremento no equilíbrio postural e na força muscular inspiratória, porém sem implicações na força muscular periférica, medo de queda e mobilidade funcional de cardiopatas inseridos na RC - Fase II.

PALAVRAS-CHAVE: Exercícios Respiratórios; Doença das Coronárias; Reabilitação Cardíaca; Força Muscular; Equilíbrio Postural.

\section{ABSTRACT}

Objective: To evaluate the effects of moderate-to-high intensity of inspiratory muscle training (IMT) associated with cardiac rehabilitation (CR) on postural balance, inspiratory and peripheral muscle strength, fear of falling and functional mobility of patients in Phase II of CR. Methods: This is a quasi-experimental design study. Previously to the initial assessments, 12 subjects were randomized into an inspiratory muscle training group (GTMI, $n=7$ ) with moderate to high intensity training load or a Sham inspiratory muscle training group (GTMIS, $n=5$ ) with a minimum load maintained constant. The following outcomes were assessed: postural balance (Berg Balance Scale-BBS and Balance Evaluation Systems Test-BESTest); inspiratory muscle strength (maximal inspiratory pressure-MIP); peripheral muscle strength (test of a maximum repetition-1RM); self-perceived confidence (Activities-specific Balance Confidence-ABC); functional mobility (Timed Up and Go-TUG and 6-meter gait speed test). The training protocol consisted of IMT, aerobic and resistance exercise for 12 weeks, with two weekly sessions. Results: The findings of this pilot study demonstrated that after intervention, in the intra-group comparison, the GTMI showed an increase in the BESTest score (pre 94.7 points vs post 101 points, $p=<0.001$ ), in MIP expressed in absolute values (pre $81.43 \mathrm{cmH} 20$ vs post $105.86 \mathrm{cmH} 20$, $p=0.006$ ) and percentage of predicted (pre $89.37 \%$ vs post $115.10 \%, p=0.002$ ), as well as the reduction in TUG time (pre $8,04 \mathrm{~s}$ vs post $6.54 \mathrm{~s}, \mathrm{p}=0.009$ ). In the comparison between the groups, there was an increase in the BESTest score $(p=0.049)$ and in the MIP expressed in absolute values $(p=0.041)$ and percentage of predicted $(p=0.017)$ in favor of GTMI. However, with no significant difference for the other outcomes assessed. Conclusion: The results of this pilot study suggest that the moderate-to-high intensity of IMT combined with CR provided an increase in postural balance and inspiratory muscle strength, but without implications for peripheral muscle strength, fear of falling and functional mobility of cardiac patients inserted in CR - Phase II. 


\section{INTRODUÇÃO}

A cirurgia cardíaca é considerada o procedimento de escolha visando redução de sintomas e mortalidade conforme a condição clínica do paciente. Porém, trata-se de um procedimento complexo, com diversas complicações clínicas e funcionais, acometendo diferentes sistemas, dentre eles o sistema muscular esquelético, culminando assim, na redução da força muscular respiratória e periférica¹. Em decorrência dessas complicações, o equilíbrio pode ser comprometido, uma vez que alterações na função física vêm sendo relacionadas ao risco de quedas, dependência física e pior saúde autorreferida².

Em indivíduos submetidos à cirurgia cardíaca, a proteólise muscular é um fator determinante para o declínio funcional no pós operatório, cuja etiologia está associada a um estado de hipercatabolismo propiciado pelo aumento na produção de citocinas inflamatórias ${ }^{3}$.

Além disso, também pode ser observada a presença de disfunção muscular diafragmática ${ }^{4}$. Considerando a importante função do diafragma na estabilidade da coluna lombar, através da conexão de suas fibras crurais com as vértebras ${ }^{5}$, o comprometimento da função muscular, acarreta uma deficiente ação estabilizadora, corroborando na redução do equilíbrio ${ }^{4,5}$. Nesse sentido, sugere-se que a redução da função muscular inspiratória ${ }^{5}$ associada à demanda inspiratória aumentada6, poderia culminar na inibição da ação estabilizadora do diafragma para com a coluna ${ }^{4,6}$.

Em decorrência destas complicações advindas do procedimento cirúrgico, é imprescindível analisar a força muscular respiratória e periférica destes pacientes, a fim de compreender a magnitude e suas implicações funcionais ${ }^{1}$, principalmente sobre o equilíbrio postural. Nesse cenário clínico, as alterações de equilíbrio requerem atenção, principalmente em virtude de que tais déficits podem comprometer a execução do treinamento físico nos programas de $\mathrm{RC}$, resultando até mesmo em quedas. Entretanto, esta variável raramente é mensurada em programas de RC.

Diante deste contexto, o TMl vem sendo incluído nos programas de RC, por conferir benefícios adicionais à reabilitação destes pacientes. Um recente ensaio clínico randomizado demonstrou efeitos terapêuticos importantes viabilizados por tal recurso, principalmente na força muscular inspiratória evidenciada pelo incremento na pressão inspiratória máxima?.

Diversos estudos vêm investigando os benefícios propiciados pelo TMI no equilíbrio postural em indivíduos saudáveis ${ }^{6,8}$ e não saudáveis ${ }^{9,10}$. Como no estudo conduzido por Bosnak-Guclu et al. ${ }^{9}$, em indivíduos com insuficiência cardíaca congestiva (ICC), demonstrando que a aplicação de um protocolo de TMI gerou melhora na força muscular periférica e no equilíbrio funcional.

Frente ao exposto, este estudo piloto teve como objetivo investigar os efeitos do TMI de moderada a alta intensidade, associado à reabilitação cardíaca $(\mathrm{RC})$, no equilíbrio postural, força muscular inspiratória e periférica, medo de queda e mobilidade funcional de pacientes inseridos em um programa de RC - Fase II. 


\section{MÉTODO}

\section{Design do estudo}

Trata-se de um estudo piloto de delineamento do tipo quase-experimental, realizado na Unidade de Reabilitação do Hospital Universitário de Santa Maria (HUSM), durante o período de junho de 2019 a março de 2020. Inicialmente foram avaliados 20 indivíduos candidatos a participar do Programa de Reabilitação Cardíaca - Revicardio, vinculado ao Serviço de Cardiologia do HUSM.

\section{Participantes}

Os critérios de elegibilidade incluíram idade mínima de 55 anos, ambos os sexos, recrutados da lista de espera para a Fase II da RC do hospital supracitado, submetidos à CRM,

troca valvar ou angioplastia coronária (com implante de stent), com anuência da equipe médica para prática de exercícios físicos e disponibilidade de comparecer ao treinamento físico. Foram excluídos os pacientes com hipertensão arterial não controlada (Pressão Arterial Sistólica (PAS) > 190 e/ou Pressão Arterial Diastólica (PAD) > 120), angina instável, insuficiência cardíaca descompensada, asma, bloqueio atrioventricular de $3^{\circ} \mathrm{grau}$ (sem e com marcapasso), arritmia cardíaca complexa, insuficiência ou estenose mitral ou aórtica graves sem tratamento adequado, problemas ortopédicos ou neurológicos graves, labirintite, diabetes mellitus descontrolada e inaptidão cognitiva.

O estudo foi aprovado pelo Comitê de Ética em Pesquisa com Seres Humanos da UFSM (CAAE 85774518.3.0000.5346). Todos os sujeitos assinaram o termo de consentimento livre e esclarecido previamente às avaliações.

\section{Randomização}

Inicialmente os sujeitos incluídos no estudo foram randomizados em dois grupos: grupo TMI de moderada a alta intensidade (GTMI) e grupo TMI Sham carga mantida constante (GTMIS). Ambos os grupos realizaram o mesmo protocolo de RC.

Previamente às avaliações iniciais, o processo da randomização foi realizado por um colaborador externo ao estudo, através de uma ordem aleatória de alocação, utilizando os números 1 e 2 para designar os sujeitos do GTMI e do GTMIS respectivamente, através do software Random Number Generator Generator (Pro v2.00, Segobit, Issaquah, WA, EUA).

\section{Avaliações}

Todos os pacientes foram submetidos a três dias de avaliações, com um intervalo mínimo de 48 horas entre 
elas. No primeiro dia foram realizadas as seguintes avaliações: mobilidade funcional (Timed Up and Go-TUG) e equilíbrio postural (Berg Balance Scale-BBS). No segundo dia foram avaliadas: mobilidade funcional (teste de velocidade da marcha de seis metros-TVM6), avaliação da confiança auto-percebida (medo de queda) na realização de atividades sem perder 0 equilíbrio (Activities-Specific Balance Confidence - ABC) e avaliação do equilíbrio postural (Balance Evaluation Systems Test-BESTest). Por fim, no terceiro dia, realizou-se a avaliação da força muscular inspiratória (pressão inspiratória máxima-PImáx) e da força muscular periférica de membros inferiores (teste de uma repetição máxima-1RM). Cabe destacar que todas as avaliações foram realizadas por avaliadores previamente treinados.

\section{Equilíbrio postural}

O desfecho equilíbrio postural foi avaliado através da Berg Balance Scale (BBS) e do Balance Evaluation Systems Test (BESTest). A escala BBS avalia o equilíbrio funcional em 14 itens comuns às atividades de vida diária. 0 escore máximo é de 56 e o mínimo é 0 , de modo que cada item apresenta uma escala decrescente de cinco opções, a qual varia de 0 a 4 pontos $^{11}$. $O$ escore foi pontuado conforme o estudo de Blum e Korner-Bitensky ${ }^{12}$.

O BESTest avalia 6 sistemas básicos relacionados ao equilíbrio: limitações biomecânicas, limites de estabilidade, respostas posturais, ajustes posturais antecipatórios, orientação sensorial e equilíbrio dinâmico durante a marcha e efeitos cognitivos. Sendo composta por 27 atividades e 6 subgrupos, representando um total de 36 itens. Cada item é pontuado numa escala decrescente de 4 níveis a partir de 0 (pior desempenho) para 3 (melhor desempenho) ${ }^{13}$. 0 avaliador realizou treinamento prévio conforme os vídeos e instruções disponibilizadas pelo instrumento. Os valores obtidos foram comparados aos valores normativos propostos por O'Hoski et al ${ }^{14}$.

\section{Força muscular inspiratória}

Para aferir a força muscular inspiratória, foi utilizada a medida da PImáx, obtida por um manovacuômetro digital MVD $300 \circledR$ (GlobalMed, Porto Alegre, Brasil). Assim, foi solicitado um esforço inspiratório máximo a partir do volume residual (VR). $O$ teste foi composto de pelo menos, 6 manobras com um intervalo de 1 minuto entre cada medida. Considerou-se completa diante da realização de 3 manobras aceitáveis e reprodutíveis (variação inferior a 10\%). 0 maior valor de PImáx foi registrado ${ }^{15}$. Um único avaliador realizou as mensurações de todos os indivíduos, como forma de padronizar os comandos verbais de estímulo. Para análise dos resultados, foi considerado o valor predito de acordo com a equação proposta por Pessoa et al. ${ }^{16}$.

\section{Força muscular periférica}

A mensuração da força dos membros inferiores (MMII) ocorreu através da realização do teste de uma repetição 
máxima (1RM), de acordo com as recomendações das diretrizes do American College of Sports Medicine (ACSM) ${ }^{17}$.

\section{Confiança auto-percebida}

A medida da confiança auto-percebida que permite avaliar o medo de queda ocorreu por meio da escala ABC, na qual estima numericamente o nível de confiança para realização de atividades específicas sem que haja desequilíbrio ou instabilidade. Este instrumento é constituído por 16 itens, sendo aplicado na forma de entrevista. Cada item mensura o nível de confiança na realização de uma tarefa específica, foi solicitado aos pacientes que atribuíssem valores de $0 \%$ (sem confiança) a 100\% (totalmente confiante). O escore total da escala ABC foi obtido pelo somatório das avaliações (01,600), sendo dividido pelo número total de itens ${ }^{18}$. Para análise do resultado obtido, foi utilizada a classificação posposta por Myers et al. ${ }^{19}$.

\section{Mobilidade funcional}

Com a finalidade de mensurar a mobilidade funcional foi realizado o TUG, o teste consistiu em uma caminhada, na qual o indivíduo foi orientado a percorrer a distância demarcada de 3 metros em ritmo habitual e de forma segura. A avaliação iniciou após o acionamento do cronômetro no momento em que o paciente se levantou da cadeira (assento com altura de $46 \mathrm{~cm}$ ), e mediante o posicionamento do dorso do paciente no encosto da cadeira, o cronômetro foi encerrado, e assim, foi mensurado o tempo gasto em segundos para sua execução. 0 teste foi realizado uma vez para efeito de familiarização ${ }^{20}$, seguida assim, de mais duas medidas no mesmo dia, com intervalo de 4 minutos de descanso para obter a média final. Valores superiores a 8,47s foram considerados baixo desempenho, conforme proposto por Bellet et al. ${ }^{21}$.

O TVM6, também foi utilizado para avaliar a mobilidade funcional, no qual foi solicitado que o indivíduo caminhasse por um percurso demarcado de 10 metros, o mais rápido possível, mas sem correr. O sujeito realizou o percurso uma vez para familiarização, e assim, realizou mais 3 medidas, com intervalo de descanso de um 1 entre elas. Para o cálculo da velocidade da marcha foi considerado a distância entre o segundo e oitavo metro, sendo obtido através da divisão dos seis metros de percurso pelo tempo médio das 3 medidas validadas ${ }^{22}$. Os valores obtidos foram comparados aos normativos propostos por Steffen et al. ${ }^{23}$.

\section{Programa de reabilitação cardíaca}

O treinamento físico foi composto por exercício aeróbico e de resistência durante o período de 12 semanas, com 2 sessões semanais. Sendo o exercício aeróbico realizado em esteira ergométrica (Inbramed, ATL - 10200, RS, BR), por um período de 30 minutos, a frequência cardíaca $(\mathrm{FC})$ de treinamento foi calculada pela diferença entre a $\mathrm{FC}$ máxima 
e a FC de repouso obtida por meio de teste ergométrico, com intensidade ajustada de 55 a $65 \%$ da FC de treinamento. Assim como, a Escala de Borg modificada foi utilizada para mensurar o nível de esforço percebido atingindo valor de 4 a 6 , em uma variação de 0 a $10^{24}$.

Os exercícios de resistência para membros superiores (músculos bíceps braquial, tríceps braquial, deltoide e peitoral) e membros inferiores (quadríceps femoral, adutores e abdutores de quadril, gastrocnêmico e sóleo) foram realizados com halteres, caneleiras ou bandas elásticas, sendo executadas 3 séries de 10 repetições por grupo muscular, com intervalo de 30 segundos de descanso entre as séries. A intensidade foi ajustada para 50\% do teste 1RM e a duração total foi de 20 minutos $^{24}$. Além de exercícios de flexibilidade para os membros superiores e inferiores, antes e após o treinamento. Ressalta-se que constantemente foi realizada a monitorização dos sinais vitais: pressão arterial (PA), FC, saturação periférica de oxigênio (SpO2), frequência respiratória (FR), sensação de dispneia (SD), sensação de fadiga nos membros inferiores (SFMMII).

\section{Treinamento muscular inspiratório}

O TMI foi realizado previamente ao treinamento físico da RC, através de um dispositivo resistor inspiratório de carga pressórica linear PowerBreathe® (Medic Plus, SP, BR), que permite cargas de até $78 \mathrm{cmH} 20$. Cuja duração da intervenção foi de 12 semanas, sendo duas sessões semanais, sob supervisão direta de um fisioterapeuta. O paciente foi instruído a sentar-se confortavelmente, fazendo uso de um clip nasal, com membros superiores apoiados sobre a mesa e uma das mãos segurando o equipamento acoplado a boca através de um bucal, firmemente envolvido pelos lábios. Durante o treinamento os pacientes foram instruídos a manter o padrão respiratório diafragmático.

O protocolo de treinamento consistiu em 5 séries com 10 repetições cada, durante o descanso, a FC, SpO2 e SD eram registradas. Foi adotado um intervalo de descanso de pelo menos 1 ou de acordo com o feedback fornecido pelos pacientes através da escala de Borg modificada. Ao término da intervenção, os sinais vitais previamente mesurados eram novamente aferidos.

Abaixo são expostos separadamente os protocolos de TMl empregados em cada grupo. Conforme estudo prévio de Dos Santos et al. ${ }^{7}$.

\section{Grupo treinamento muscular inspiratório de moderada a alta intensidade (GTMI)}

O GTMI realizou incremento semanal de carga, associado ao treinamento físico composto de exercícios aeróbicos e de resistência. A carga inicial utilizada no aparelho foi de 50\% da PImáx, nas duas primeiras semanas, para que houvesse um período de adaptação. Após, o incremento da carga deu-se da seguinte maneira: 55\% da PImáx na terceira semana, $60 \%$ da PImáx na quarta semana, $65 \%$ da PImáx na quinta semana, $70 \%$ da PImáx na sexta semana, 
75\% da PImáx na sétima semana e 80\% da PImáx na oitava semana. Após a oitava semana, até a décima segunda, foi realizada nova manovacuometria semanal para manter $80 \%$ da nova PImáx.

\section{Grupo treinamento muscular inspiratório Sham (GTMIS)}

O GTMIS realizou o treinamento com a carga mínima do aparelho, $9 \mathrm{cmH} 20$, mantida constante, durante as 12 semanas do estudo, em consonância ao treinamento físico composto de exercícios aeróbicos e de resistência. Ressaltase que os pacientes receberam as mesmas instruções citadas anteriormente, bem como, a monitorização dos sinais vitais.

\section{Análise estatística}

Os dados foram analisados no software estatístico GraphPad Prism 5 (GraphPad Software Inc., San Diego, CA, EUA). A normalidade das variáveis foi avaliada pelo teste de Shapiro-Wilk. As variáveis contínuas são apresentadas na forma de média, desvio padrão e intervalo de confiança de 95\% (IC 95\%) e as variáveis categóricas descritas em frequências absolutas e porcentagens. $O$ teste $T$ de Student para amostras pareadas foi utilizado para comparar os resultados antes e após a intervenção intragrupo. A ANOVA two-way foi realizada para a comparação dos resultados entre os grupos. Além disso, uma análise de regressão linear simples foi realizada entre o escore total obtido no BESTest (variável dependente) e a PImáx (variável independente) sendo considerados os momentos pré e pós intervenção. 0 nível de significância foi estabelecido em $5 \%(p \leq 0,05)$.

\section{RESULTADO}

Inicialmente foram avaliados 20 indivíduos para elegibilidade, 1 indivíduo foi excluído devido a faixa etária, assim, 19 sujeitos foram randomizados em GTMI $(n=10)$ e GTMIS $(n=9)$. Destes, 7 pacientes não finalizaram o programa de RC: 3 indivíduos do GTMI, e 4 indivíduos do GTMIS. Assim, 12 pacientes completaram o programa de RC e foram inseridos na análise, sendo no GTMI ( $n=7)$ e no GTMIS ( $n=5)$, conforme apresentado no fluxograma do estudo (Figura 1). 
Figura 1. Fluxograma do estudo

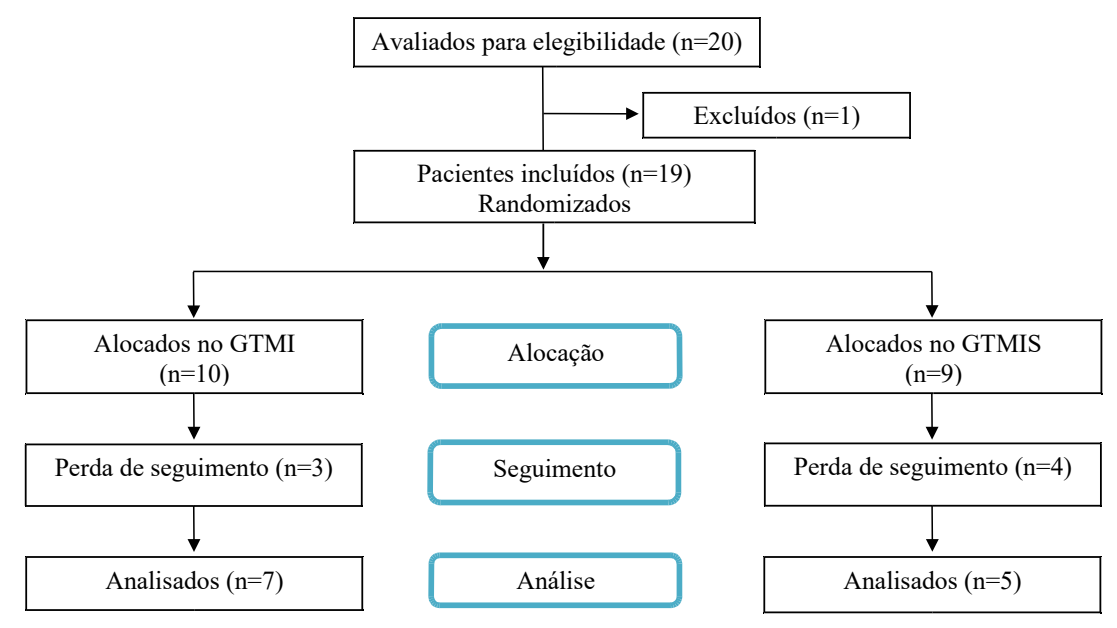

GTMI: grupo treinamento muscular inspiratório de moderada a alta intensidade associado à RC (treinamento aeróbico e resistido); GTMIS: grupo treinamento muscular inspiratório Sham com carga mínima constante, associado à RC (treinamento aeróbico e resistido).

A Tabela 1 demonstra que os grupos eram semelhantes quanto às características antropométricas, clínicas e as variáveis de desfecho na linha de base.

Tabela 1. Características antropométricas, clínicas, fatores de risco, medicamentos e as variáveis de desfecho na linha de base.

\begin{tabular}{lccc}
\hline Características & GTMI ( $\mathrm{n}=7)$ & GTMIS ( $\mathrm{n}=5)$ & p-valor \\
\hline Antropométricas & & & \\
Sexo masculino, $\mathrm{n}(\%)$ & $6(85,7)$ & $4(80,0)$ & 0,815 \\
Idade (anos) & $57,4 \pm 9,0$ & $58 \pm 7,6$ & 0,911 \\
IMC $\left(\mathrm{Kg} / \mathrm{m}^{2}\right)$ & $28,5 \pm 3,1$ & $26,6 \pm 4,1$ & 0,372 \\
Clínicas & & & \\
Procedimento, $\mathrm{n}(\%)$ & & & 0,599 \\
$\quad$ CRM & $4(57,1)$ & $1(20,0)$ & - \\
$\quad 1(14,3)$ & $2(40,0)$ & - \\
$\quad$ Troca valvar & $2(28,6)$ & $2(40,0)$ & - \\
$\quad$ Angioplastia & $60,4 \pm 6,8$ & $60,4 \pm 10,5$ & 0,995 \\
FEVE (\%) & $4,5 \pm 1,5$ & $4,6 \pm 2,1$ & 0,930 \\
Tempo pós procedimento (meses) & & & \\
Fatores de risco, $\mathrm{n}(\%)$ & $2(28,6)$ & $3(60,0)$ & 0,319 \\
Tabagismo pregresso & $3(42,8)$ & $1(20,0)$ & 0,454 \\
Tabagismo atual & $1(14,3)$ & $1(20,0)$ & 0,815 \\
Dislipidemia & $1(14,3)$ & $1(20,0)$ & 0,815 \\
Obesidade & $2(28,6)$ & $2(40,0)$ & 0,711 \\
Diabetes mellitus & $7(100,0)$ & $3(60,0)$ & 0,356 \\
HAS & & $3(80,0)$ & 0,815 \\
Medicamentos, $\mathrm{n}(\%)$ & $6(85,7)$ & $2(40,0)$ & 0,355 \\
AINEs & $1(14,3)$ & $3(60,0)$ & 0,290 \\
Antiplaquetário & $7(100,0)$ & & \\
Estatina & & & \\
\end{tabular}




\begin{tabular}{lccc} 
Diurético & $1(14,3)$ & - & - \\
Anticoagulante & - & $1(20,0)$ & - \\
IECA & $4(57,1)$ & $2(40,0)$ & 0,599 \\
Beta-bloqueador & $7(100,0)$ & $4(80,0)$ & 0,815 \\
Variáveis de desfecho & & & \\
BESTest (escore total) & $94,7 \pm 4,57$ & $96,8 \pm 6,83$ & 0,538 \\
BBS (pontos) & $54,85 \pm 1,34$ & $53,4 \pm 3,64$ & 0,726 \\
TUG (segundos) & $8,04 \pm 1,69$ & $7,4 \pm 1,75$ & 0,236 \\
ABC (escore total) & $72,49 \pm 28,17$ & $85,37 \pm 27,75$ & 0,450 \\
TVM6 (m/s) & $1,63 \pm 0,32$ & $1,78 \pm 0,28$ & 0,406 \\
PImáx (cmH & $81,0)$ & $74 \pm 16,67$ & 0,436 \\
1 RM $(\mathrm{Kg})$ & $8,5 \pm 1,6$ & $7,5 \pm 1,5$ & 0,300 \\
\hline
\end{tabular}

Os dados são expressos como média (DP) ou frequência (\%). Abreviações: GTMI: grupo treinamento muscular inspiratório de moderada a alta intensidade; GTMIS: grupo treinamento muscular inspiratório Sham; IMC: índice de massa corporal; CRM: cirurgia de revascularização do miocárdio; FEVE: fração de ejeção do ventrículo esquerdo; HAS: hipertensão arterial sistêmica; AINEs: anti-inflamatórios não esteroides; IECA: inibidores da enzima conversora da angiotensina; BESTest: Balance Evaluation Systems Test; BBS: Berg Balance Scale; TUG: Timed up and Go; ABC: Activities-Specific Balance Confidence; TVM6: Teste de velocidade de marcha de 6 metros; PImáx: pressão inspiratória máxima; 1 RM: uma repetição máxima. * Significância estatística p<0,05.

Considerando a comparação intragrupo, o GTMI apresentou incremento significativo no escore total do BESTest (pré 94,7 pontos vs pós 101 pontos, p<0,001) e na PImáx, expressos pelos valores absolutos (pré 81,43 cmH2O vs pós $105,86 \mathrm{cmH} 2 \mathrm{O}, \mathrm{p}=0,006$ ) e porcentagem atingida quanto ao predito (pré $89,37 \%$ vs pós $115,10 \%, p=0,002$ ), assim como, redução significativa no TUG (pré 8,04 s vs pós 6,54 s, p=0,009) após intervenção (Tabela 2). 
Tabela 2. Comparação intra e entre os grupos para equilíbrio e demais desfechos avaliados.

\begin{tabular}{|c|c|c|c|c|c|c|c|c|c|c|}
\hline \multirow[b]{3}{*}{ Variáveis } & \multicolumn{2}{|c|}{ GTMI $(n=7)$} & \multirow[b]{3}{*}{$\begin{array}{c}\text { Diferença média (IC } \\
95 \%)\end{array}$} & \multirow[b]{3}{*}{$\begin{array}{c}p- \\
\text { valor }^{\mathrm{a}}\end{array}$} & \multicolumn{2}{|c|}{ GTMIS ( $n=5)$} & \multirow[b]{3}{*}{$\begin{array}{c}\text { Diferença média (IC } \\
95 \%)\end{array}$} & \multirow[b]{3}{*}{$\begin{array}{c}p- \\
\text { valor }^{\mathrm{a}}\end{array}$} & \multirow{2}{*}{\multicolumn{2}{|c|}{$\begin{array}{c}\text { Efeito da intervenção } \\
\text { Grupo vs tempo }\end{array}$}} \\
\hline & \multirow{2}{*}{$\frac{\text { Pré }}{\text { Média } \pm \text { DP }}$} & \multirow{2}{*}{$\frac{\text { Pós }}{\text { Média } \pm \text { DP }}$} & & & \multirow{2}{*}{$\frac{\text { Pré }}{\text { Média } \pm \text { DP }}$} & \multirow{2}{*}{$\frac{\text { Pós }}{\text { Média } \pm \text { DP }}$} & & & & \\
\hline & & & & & & & & & $\begin{array}{l}\text { Diferença entre } \\
\text { grupos (IC 95\%) }\end{array}$ & $p$-valor ${ }^{b}$ \\
\hline \multicolumn{11}{|c|}{ Força muscular periférica dos MMII } \\
\hline $1 \mathrm{RM}(\mathrm{Kg})$ & $8,5 \pm 1,6$ & $9,1 \pm 3,95$ & $0,6(-4,22$ a 5,37$)$ & 0,780 & $7,5 \pm 1,5$ & $8,2 \pm 2,25$ & $0,70(-0,66$ a 2,06$)$ & 0,226 & $-0,9(-4,6$ a 2,86$)$ & 0,958 \\
\hline PImáx. $\left(\mathrm{cmH}_{2} \mathrm{O}\right)$ & $81,43 \pm 15,92$ & $105,86 \pm 25,76$ & $24,43(9,77$ a 39,09$)$ & $0,006^{*}$ & $74 \pm 16,67$ & $78,6 \pm 19,51$ & $4,6(-10,36$ a 19,56$)$ & 0,441 & $-27,26(-55,55$ a 1,03$)$ & $0,041^{*}$ \\
\hline PImáx. (\% pred. $)^{1}$ & $89,37 \pm 13,16$ & $115,10 \pm 15,04$ & $25,73(13,52$ a 37,92$)$ & $0,002^{*}$ & $88,45 \pm 27,69$ & $91,77 \pm 20,35$ & $3,32(-13,67$ a 20,32$)$ & 0,615 & $-23,32(-50,10$ a 3,45$)$ & $0,017^{*}$ \\
\hline \multicolumn{11}{|l|}{ Mobilidade funcional } \\
\hline TUG (segundos) & $8,04 \pm 1,69$ & $6,54 \pm 1,24$ & $-1,5(-2,47$ a $-0,51)$ & $0,009^{*}$ & $7,4 \pm 1,75$ & $6,82 \pm 1,14$ & $-0,58(-1,42$ a 0,26$)$ & 0,127 & $0,28(1,83$ a 2,38$)$ & 0,123 \\
\hline TVM6 (m/s) & $1,63 \pm 0,32$ & $1,71 \pm 0,36$ & $0,08(-0,07$ a 0,22$)$ & 0,247 & $1,78 \pm 0,28$ & $1,84 \pm 0,33$ & $0,06(-0,13$ a 0,25$)$ & 0,431 & $0,13(-0,35$ a 0,60$)$ & 0,855 \\
\hline \multicolumn{11}{|l|}{ Equilíbrio postural } \\
\hline BBS (pontos) & $54,85 \pm 1,34$ & $55 \pm 1,73$ & $0,15(-0,49$ a 0,78$)$ & 0,603 & $53,4 \pm 3,64$ & $55 \pm 1,73$ & $1,6(-1,5$ a 4,7$)$ & 0,603 & $0(-2,97$ a 2,97$)$ & 0,168 \\
\hline BESTest (escore total) & $94,7 \pm 4,57$ & $101 \pm 3,65$ & $6,3(3,91$ a 8,65$)$ & $<0,001^{*}$ & $96,8 \pm 6,83$ & $98,2 \pm 6,45$ & $1,4(-4,84$ a 7,64$)$ & 0,567 & $-2,8(-10,3$ a 4,70$)$ & $0,049^{*}$ \\
\hline \multicolumn{11}{|c|}{ Confiança auto-percebida } \\
\hline ABC (escore total) & $72,49 \pm 28,17$ & $85,08 \pm 13,25$ & $12,59(-5,75$ a 30,93$)$ & 0,144 & $85,37 \pm 27,75$ & $91,12 \pm 13,73$ & $5,75(-27,54$ a 39,04$)$ & 0,656 & $6,04(-25,13$ a 37,21$)$ & 0,621 \\
\hline
\end{tabular}

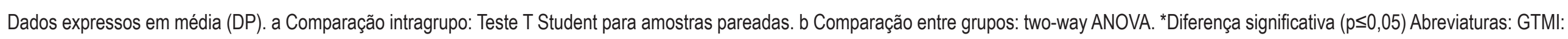

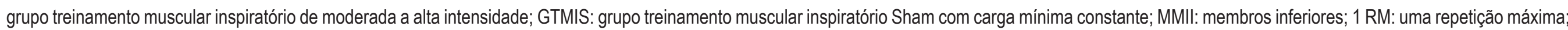

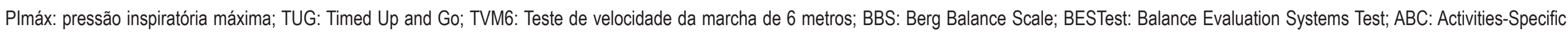
Balance Confidence; 3 Equação de Pessoa et al. 
Ainda na comparação intragrupo, a Tabela 3 demonstrou que o GTMI apresentou aumento significativo no percentual obtido em duas seções do BESTest, limitações biomecânicas (pré $79,04 \%$ vs pós $86,66 \%$, $p=0,047$ ) e reatividade (pré $94,44 \%$ vs pós $100 \%$, $p=0,038$ ) após a intervenção.

Tabela 3. Comparação intra e entre os grupos sobre o equilíbrio postural expressos em porcentagem do escore geral e de cada uma das seções de desempenho obtidas no BESTest.

\begin{tabular}{|c|c|c|c|c|c|c|c|c|c|c|}
\hline & \multicolumn{2}{|c|}{ GTMI $(n=7)$} & \multicolumn{4}{|c|}{ GTMIS $(n=5)$} & & & \multicolumn{2}{|c|}{$\begin{array}{c}\text { Efeito da interação (grupo } x \\
\text { tempo) }\end{array}$} \\
\hline & Pré & Pós & & & Pré & Pós & & & & \\
\hline Variáveis & Média \pm DP & Média \pm DP & $\begin{array}{l}\text { Diferença média } \\
\text { (IC 95\%) }\end{array}$ & $\begin{array}{c}p- \\
\text { valor }^{\mathrm{a}}\end{array}$ & Média \pm DP & Média \pm DP & $\begin{array}{l}\text { Diferença média } \\
\text { (IC 95\%) }\end{array}$ & $\begin{array}{c}p- \\
\text { valor }^{a}\end{array}$ & $\begin{array}{l}\text { Diferença entre } \\
\text { grupos (IC 95\%) }\end{array}$ & $\begin{array}{c}p- \\
\text { valor }^{b}\end{array}$ \\
\hline BESTest, \% & $87,69 \pm 4,23$ & $93,47 \pm 3,41$ & $5,78(3,63$ a 7,93$)$ & $<0,001^{*}$ & $89,62 \pm 6,32$ & $90,92 \pm 5,98$ & $1,3(-4,48$ a 7,07$)$ & 0,568 & $-2,55(-9,51$ a 4,4$)$ & $0,049^{*}$ \\
\hline Limitações biomecânicas, \% & $79,04 \pm 12,42$ & $86,66 \pm 12,17$ & $7,62(0,13$ a 15,11$)$ & $0,047^{*}$ & $79,99 \pm 10,54$ & $82,66 \pm 10,11$ & $2,67(-18,11$ a 23,44$)$ & 0,739 & $-4(-20,39$ a 12,39$)$ & 0,507 \\
\hline Limites de estabilidade/ & $80,27 \pm 9,29$ & $88,43 \pm 4,64$ & $8,15(-0,54$ a 16,86$)$ & 0,061 & $86,66 \pm 9,15$ & $88,56 \pm 9,87$ & $1,90(-13,51$ a 17,32$)$ & 0,749 & $0,13(-11,62$ a 11,90$)$ & 0,342 \\
\hline \multicolumn{11}{|l|}{ Verticalidade } \\
\hline Transições/antecipação & $88,88 \pm 5,55$ & $93,98 \pm 6,18$ & $5,1(-2,58$ a 12,79$)$ & 0,155 & $87,77 \pm 18,59$ & $89,99 \pm 11,38$ & $2,22(-8,24$ a 12,68) & 0,587 & $-3,99(-19,26$ a 11,28$)$ & 0,569 \\
\hline Reatividade & $94,44 \pm 5,56$ & $100 \pm 0$ & $5,56(0,41$ a 10,7$)$ & $0,038^{*}$ & $100 \pm 0$ & $100 \pm 0$ & - & - & - & - \\
\hline Orientação sensorial & $100 \pm 0$ & $99,04 \pm 2,52$ & $-0,95(-3,28$ a 1,37$)$ & 0,355 & $100 \pm 0$ & $100 \pm 0$ & - & - & $0,95(-1,00$ a 2,91$)$ & 0,424 \\
\hline
\end{tabular}

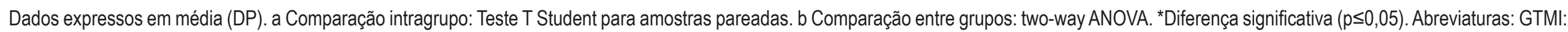
grupo treinamento muscular inspiratório de moderada a alta intensidade; GTMIS: grupo treinamento muscular inspiratório Sham; BESTest: Balance Evaluation Systems Test.

De acordo com a Figura 2A e 2B, na comparação entre os grupos, houve um aumento significativo no escore total do BESTest ( $p=0,049)$, na PImáx nos valores absolutos $(p=0,041)$, assim como na porcentagem atingida do predito $(p=0,017)$ à favor do GTMI, em comparação ao GTMIS. 
Figura 2. Comparação intragrupo e entre os grupos para os desfechos equilíbrio postural e força muscular inspiratória.

A

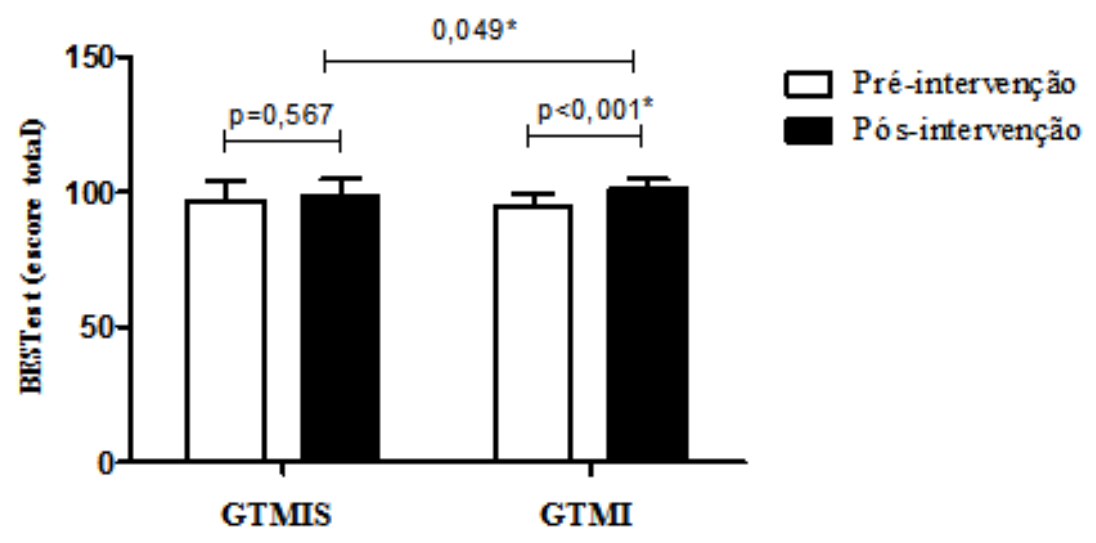

B

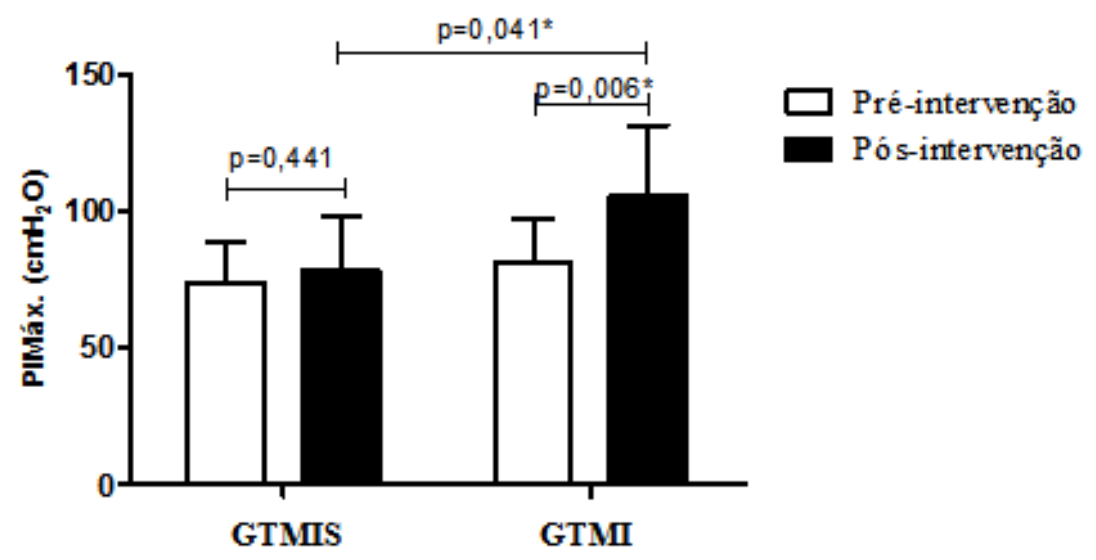

Comparação intragrupo: Teste T Student para amostras pareadas. b Comparação entre os grupos: ANOVA de duas vias. *Diferença significativa ( $p \leq 0,05)$. Abreviaturas: GTMI: grupo treinamento muscular inspiratório de moderada a alta intensidade; GTMIS: grupo treinamento muscular inspiratório Sham; BESTest: Balance Evaluation Systems Test;PImáx: pressão inspiratória máxima.

$\mathrm{Na}$ análise de regressão linear, a PImáx não demonstrou exercer influência na pontuação obtida no BESTest nos momentos pré $\mathrm{RC}(\beta=0,148 ;$ IC $95 \%=-0,08$ a 0,$37 ; p=0,174)$ ou pós $\mathrm{RC}(\beta=0,099 ;$ IC $95 \%=-0,01$ a 0,$21 ; p=0,077)$, conforme demonstrado na Tabela 4.

Tabela 4. Análise de regressão linear entre equilíbrio postural e força muscular inspiratória nos momentos avaliados.

\begin{tabular}{llllllll}
\hline Variável dependente & Variável independente & $\mathbf{R}^{2}$ & $\mathbf{R}^{2}$ ajustado & Coeficiente & $\boldsymbol{\beta}$ & IC $95 \%$ & p valor \\
\hline Pré & BESTest (pontuação) & PImáx $\left(\mathrm{cmH}_{2} \mathrm{O}\right)$ & 0,18 & 0,09 & 0,148 & $-0,08$ a 0,37 & 0,174 \\
Pós & BESTest (pontuação) & PImáx $\left(\mathrm{cmH}_{2} \mathrm{O}\right)$ & 0,28 & 0,21 & 0,099 & $-0,01$ a 0,21 & 0,077 \\
\hline
\end{tabular}

PImáx: pressão inspiratória máxima. IC: Intervalo de confiança. Significância estatística $p<0,05$. Abreviaturas: BESTest: Balance Evaluation Systems Test;PImáx: pressão inspiratória máxima. 


\section{DISCUSSÃO}

Os achados deste estudo piloto demonstraram que a adição de um protocolo de TMI de moderada a alta intensidade ao treinamento aeróbico e resistido em um programa de RC (Fase II) foi capaz de proporcionar incremento no equilíbrio postural e na força muscular inspiratória. Entretanto, não houve implicações na força muscular periférica, medo de queda e mobilidade funcional. Considerando a análise de regressão linear, a força muscular inspiratória demonstrou não influenciar o equilíbrio postural destes sujeitos, nos momentos pré e pós RC.

Em consonância com os resultados alcançados neste estudo, Ferraro et al. ${ }^{8}$, evidenciaram que um protocolo de TMI (50\% da PImáx, 30 respirações, 2 vezes ao dia, durante 8 semanas), em idosos saudáveis, foi capaz de propiciar melhora do equilíbrio (dinâmico e reativo) e da força muscular inspiratória. É importante ressaltar que mesmo em uma amostra composta por adultos cardiopatas e com um programa de intervenção de menor frequência semanal, no presente estudo o TMI foi capaz de proporcionar incremento no equilíbrio postural e na força muscular inspiratória.

Ao encontro de nossos achados, Janssens et al. ${ }^{6}$ relataram que o TMI de alta intensidade (60\% PImáx, 2 vezes ao dia, 7 dias por semana, durante 8 semanas), conduzido em adultos com dor lombar inespecífica, ocasionou incremento na força muscular inspiratória. Os autores inferiram que este aumento foi, possivelmente, ocasionado por uma melhor estabilização do tronco pela participação do diafragma no controle postural, diminuindo a dependência proprioceptiva do tornozelo.

Neste sentido, os mecanismos fisiológicos que podem elucidar tais achados compreendem o fato de que 0 diafragma, músculo primário envolvido na inspiração ativa, desempenha também um importante papel no controle postural, uma vez que atua na estabilização do tronco durante a execução de tarefas que desafiam a estabilidade da coluna vertebral, bem como, é ativado na fase preparatória de movimentos que induzem instabilidade postural5. Premissa, essa corroborada por estudo conduzido em pacientes com DPOC, ao demonstrar que especialmente aqueles com fraqueza muscular inspiratória, apresentaram respostas compensatórias ao equilíbrio relacionadas à atividade do diafragma e dos músculos do tronco prejudicadas, consequentemente podendo resultar em aumento da dependência dos sinais proprioceptivos do tornozelo e redução da dependência de sinais proprioceptivos do dorso, culminando em diminuição da estabilidade postural e maior taxa de quedas ${ }^{25}$. Assim, o incremento na força muscular inspiratória pode resultar em melhora do equilíbrio postural conforme sugerido em nosso estudo piloto também de sujeitos cardiopatas.

Neste estudo, os pacientes alocados no GTMI apresentavam redução no valor da linha de base do BESTest, em comparação com o valor normativo de 95,7 pontos, proposto por O'Hoski et al. ${ }^{14}$. A partir dessa premissa, infere-se que no momento pré-intervenção os pacientes apresentaram redução do equilíbrio postural, cujo incremento significativo ocorreu somente após o uso do TMI. No entanto, ressalta-se que para a população investigada, na literatura são inexistentes 
valores de normalidade. Limitando assim, a comparação e a aplicabilidade destes valores de referência para sujeitos de outras nacionalidades e com comorbidades.

É importante destacar que em dois subitens do BESTest houve incremento significativo à favor do GTMI. Na primeira seção, limitações biomecânicas, avalia-se a qualidade da base de apoio do pé, o alinhamento postural, a funcionalidade do tornozelo e a força do quadril para ficar na posição ortostática e a capacidade de levantar-se do chão ${ }^{13}$. Restrições no sistema biomecânico limitam a capacidade de usar uma estratégia de tornozelo ou medidas compensatórias para recuperação postural ${ }^{26}$. Assim como na terceira seção, reatividade postural, que avalia a resposta à perturbação externa e ao deslocamento do centro de massa. Nessas atividades, as entradas proprioceptivas dos tornozelos, joelhos, quadris e coluna vertebral são desafiadas a manter o posicionamento ortostático ${ }^{27}$. Restrições nos ajustes posturais antecipatórios aos movimentos voluntários são dependentes da interação das áreas motoras suplementares com gânglios da base a áreas do tronco cerebral e culminam em instabilidade durante o início do passo ou movimentos rápidos ${ }^{13}$. Assim, incremento nessas duas seções têm implicações clínicas relevantes no equilíbrio postural.

O uso proprioceptivo do tornozelo é predominantemente observado em sujeitos com comprometimento da função muscular inspiratória e em indivíduos que realizam treinamento com carga inspiratória ${ }^{26}$. Nesse sentido, Janssens et al.6 sugerem que o aprimoramento da força muscular inspiratória, através do TMI, predispõe ao aumento da ponderação proprioceptiva em sujeitos com lombalgia, reduzindo a dependência exclusiva de um segmento no equilíbrio postural. Tal premissa pode justificar o aumento significativo encontrado nas seções limitações biomecânicas e reatividade postural, em nosso estudo, sugerindo que o TMI promoveu uma maior adaptação e participação proprioceptiva do diafragma no equilíbrio postural.

Em relação à mobilidade funcional, observou-se uma melhora intragrupo, expressa pela redução no tempo de execução do TUG, à favor do GTMI. Infere-se que uma possível explicação para este desfecho, seria o aumento do fluxo sanguíneo aos músculos periféricos, decorrente do incremento da força muscular inspiratória, proporcionado pelo TMI, através da atenuação do metaborreflexo dos músculos inspiratórios, contribuindo assim para a melhora do desempenho físico ${ }^{28}$. Ainda que a ativação do metaborreflexo não tenha sido investigada no presente estudo.

A velocidade da marcha, também utilizada para avaliar a mobilidade funcional, em nosso estudo não demonstrou diferença significativa após a intervenção. Tal achado pode estar associado à ausência de incremento na força muscular dos membros inferiores. Considerando o valor normativo proposto por Steffen et al. ${ }^{23}$, observou-se que apenas um paciente de cada grupo apresentou velocidade da marcha de acordo com o valor de normalidade, os demais indivíduos apresentaram redução, em ambos os momentos. É válido ressaltar que a velocidade de marcha é uma mensuração rápida e de simples inserção nos programas de RC, sendo uma importante preditora do risco de mortalidade em sujeitos com doenças cardiovasculares ${ }^{29}$. 
No que se refere à BBS, não houve diferença significativa após o programa. Este desfecho pode ser atribuído ao fato de que as atividades propostas podem não ter desafiado suficientemente a capacidade de equilíbrio destes indivíduos. Ressalta-se também que a amostra apresentou uma pontuação de $\geq 41$, o que indica um bom equilíbrio e baixo risco de quedas ${ }^{12}$. Em relação ao escore obtido na escala $A B C$, quanto ao medo de sofrer queda, também não houve diferença significativa nos grupos. Destaca-se que o nível de confiança do GTMI, na linha de base do estudo, foi classificado de acordo com Myers et al. ${ }^{19}$, quanto à capacidade física em moderada e após a intervenção como alta, mesmo sem diferença estatística significativa.

Considerando o desfecho da força muscular periférica, no presente estudo não houve incremento após a intervenção proposta. Semelhantemente, no estudo conduzido por Miozzo et al. ${ }^{30}$, um programa de 12 semanas de TMI, com protocolo semelhante ao nosso, foi incapaz de incrementar a força muscular periférica. Divergindo dos achados descritos por Bosnak-Guclu et al. ${ }^{9}$, os autores demonstraram que um protocolo de TMI (40\% da PImáx, 30 min por dia, 7 dias na semana, durante 6 semanas), gerou melhora na força muscular respiratória e periférica, assim como no equilíbrio funcional de sujeitos portadores de ICC. As possíveis explicações para esse achado conflitante podem estar relacionadas ao fato de que uma maior frequência semanal das intervenções seria necessária para ocasionar uma mudança desse desfecho.

No presente estudo, a análise de regressão demonstrou que a força muscular inspiratória não exerceu influência no equilíbrio postural de pacientes inseridos na RC. Achado semelhante foi encontrado no estudo conduzido por Zeren et al. ${ }^{10} \mathrm{em}$ sujeitos com fibrose cística. Cabe destacar que no referido estudo, mesmo sendo conduzido em uma população diferente, os autores constataram que a força muscular inspiratória não influenciou a estabilidade postural. Entretanto, os achados demonstraram que a força muscular expiratória foi um preditor independente para a estabilidade postural ${ }^{10}$. Hipotetiza-se que a ausência da relação entre a força muscular inspiratória e equilíbrio postural, encontrada no nosso estudo, pode estar relacionada ao reduzido número amostral.

Nosso estudo apresenta algumas limitações que devem ser consideradas, tais como: tamanho amostral reduzido e a ausência de um instrumento mais robusto, como o dinamômetro, para mensurar a força muscular periférica. No entanto, ao nosso ver, tais limitações não comprometeram os resultados preliminares obtidos até o presente momento, de caráter inédito por evidenciar a necessidade de avaliar, assim como implementar estratégias terapêuticas nas quais aprimorem o equilíbrio postural na população cardiopata não idosa.

\section{CONSIDERAÇÕES FINAIS}

Os resultados deste estudo piloto sugerem que o TMI de moderada a alta intensidade associado à RC propiciou 
incremento no equilíbrio postural e na força muscular inspiratória, porém sem implicações na força muscular periférica, medo de queda e mobilidade funcional de cardiopatas inseridos na reabilitação cardíaca - Fase II. Tais achados são clinicamente relevantes e inovadores, pois ratificam a aplicabilidade do TMI de moderada a alta intensidade no cenário da reabilitação cardíaca, ademais o identificam como um potencial dispositivo terapêutico com implicações no equilíbrio postural de indivíduos cardiopatas.

Adicionalmente, nosso estudo ressalta que déficits no equilíbrio postural ocorrem também em adultos cardiopatas não idosos, assim a avaliação desse desfecho em programas de RC torna-se de grande relevância, uma vez que, podem comprometer o desempenho durante os exercícios físicos.

\section{REFERÊNCIAS}

1. Menezes TC, Bassi D, Cavalcanti RC, Barros JESL, Granja KSB, Calles CAN, et al. Comparisons and correlations of pain intensity and respiratory and peripheral muscle strength in the pre- and postoperative periods of cardiac surgery. Rev Bras Ter Intensiva [internet]. 2018;30(4):479-86. doi: 10.5935/0103-507X.20180069

2. Brown RT, Diaz-Ramirez LG, Boscardin WJ, Lee SJ, Steinman MA. Functional impairment and decline in middle age: a cohort study. Ann Intern Med [internet]. 2018;167(11):761-68. doi:10.7326/M17-0496.

3. Lida Y, Yamazaki T, Arima H, Kawabe T, Yamada S. Predictors of surgery-induced muscle preotelysis in patients undergoing cardiac surgery. J Cardiol. 2016;68(6):536-41. doi: 10.1016/j.jjcc.2015.11.011.

4. Kocjan J, Gzik-Zroska B, Nowakowska K, Burkacki M, Suchon S, Michnik R, et al. Impact of diaphragm function parameters on balance maintenance. PLoS one\{internet]. 2018;13(12):1-14. https://doi.org/10.1371/journal. pone.0208697

5. Kocjan J, Adamek M, Gzik-Zroska B, Czyzewski D, Rydel M. Network of breathing. Multifunctional role of the diaphragm: a review. Adv Respir Med. 2017;85(4):224ロ232. doi: 10.5603/ARM.2017.0037.

6. Janssens L, Mcconell AK, Pijnenburg M, Claeys K, Goossens N, Lysens R, et al. Inspiratory muscle training affects proprioceptive use and low back pain. Med Sci Sports Exerc [internet]. 2015;47(1):12-19. doi: 10.1249 / MSS.0000000000000385. 
7. Dos Santos TD, Pereira SN, Portella LOC, Cardoso DM, Dal Lago P, Guarda NS, Moresco RN, Albuquerque IM. Moderate-to-high intensity inspiratory muscle training improves the effects of combined training on exercise capacity in patients after coronary artery bypass graft surgery: A randomized clinical trial. Int J Cardiol [internet]. 2019;279:40-6. doi: https://doi.org/10.1016/j.jijcard.2018.12.013

8. Ferraro FV, Gavin JP, Wainwright TW, Mcconell AK. Comparison of balance changes after inspiratory muscle or otago exercise training. PLoS ONE [internet]. 2020;15(1): e0227379. doi: https://doi. org/10.1371/journal.pone.0227379

9. Bosnak-Guclu M, Arikan H, Savci S, Inal-Ince D, Tulumen E, Aytemir K, et al. Effects of inspiratory muscle training in patients with heart failure. Respir Med [internet]. 2011;105(11):1671-81. doi: https://doi.org/10.1016/j. rmed.2011.05.001

10. Zeren M, Cakir E, Gurses HN. Effects of inspiratory muscle training on postural stability, pulmonary function and functional capacity in children with cystic fibrosis: A randomised controlled trial. Respir Med. 2019;148(189):24-30. doi: 10.1016/j.rmed.2019.01.013

11. Miyamoto ST, Lombardi I Jr, Berg KO, Ramos LR, Natour J. Brazilian version of the berg balance scale. Braz J Med Biol Res [internet]. 2004;37(9):1411-21. doi: https://doi.org/10.1590/S0100-879X2004000900017

12. Blum L, Korner-Bitensky N. Usefulness of the berg balance scale in stroke rehabilitation: a systematic review. Phys Ther. 2008;88(5):559-66. doi: 10.2522/ptj.20070205

13. Horak FB, Wrisley DM, Frank J. The balance evaluation systems test (BESTest) to differentiate balance deficits. Phys Ther [internet]. 2009;89(5):484-98. doi: 10.2522 / ptj.20080071

14. O'Hoski S, Windhip B, Herridge L, Agha T, Brooks D, Beauchamp MK, et al. Increasing the clinical utility of the BESTest, mini-BESTest, and brief-BESTest: normative values in canadian adults who are healthy and aged 50 years or 0 older. Phys Ther [internet]. 2014; 2014;94(3):334-42. doi: 10.2522 / ptj.20130104 
15. Crapo RO, Casaburi CR, Coates AL, Enright PL, Macintyre NR, Mckay RT, et al. American Thoracic Society-ATS. Committee on proficiency standards for clinical pulmonary function laboratories. ATS statement: guidelines for the six-minute walk test. Am J Respir Crit Care Med. 2002;166:111-7. doi: 10.1164 / ajrccm.166.1.at1102

16. Pessoa IMBS, Houri M, Montemezzo D, Silva LAM, Andrade AD, Parreira VF. Predictive equations for respiratory muscle strength according to international and Brazilian guidelines. Braz J Phys Ther.2014;18(5):410-418. doi: http://dx.doi.org/10.1590/bjpt-rbf.2014.0044

17. Pescatello LS, et al. ACSM's Guidelines for exercise testing and prescription. 9 ed. Filadélfia: Lippincott Williams \& Wilkins; 2014. 456 p.

18. Marques AP, Mendes YC, Taddei U, Pereira CAB, Assumpção A. brazilian-portuguese translation and cross cultural adaptation of the activities-specific balance confidence (ABC) scale. Braz J Phys Ther [internet] 2013;17(2):17078. doi: http://dx.doi.org/10.1590/S1413-35552012005000072

19. Myers AM, Fletcher PC, Myers AH, Sherk W. Discriminative and evaluative properties of the activities-specific balance confidence (ABC) Scale. J Gerontol A Biol Sci Med Sci. 1998;53(4):287-94. http://dx.doi. org/10.1093/ gerona/53A.4.M287

20. Podsiadlo D, Richardson S. The timed "Up \& Go": a test of basic functional mobility for frail elderly persons. J Am Geriatr Soc [internet]. 1991;39(2):142-8. doi: https://onlinelibrary.wiley.com/doi/abs/10.1111/j.15325415.1991. tb01616.x?sid=nlm\%3Apubmed

21. Bellet RN, Francis RL, Jacob JS, Healy KM, Bartlett HJ, Adams L, et al. Timed up and go in cardiac rehabilitation: Reliability and comparison with the 6-minute walk test. J Cardiopulm Rehabil Prev. 2013;33(2):99-105. doi: 10.1097/ HCR.0b013e3182773fae

22. Martinez BP, Batista AKMS, Ramos IR, Dantas JC, Gomes IB, Forgiarini LA Jr, et al. Viabilidade do teste de velocidade de marcha em idosos hospitalizados. J Bras Pneumol [internet]. 2016;42(3):196-202. doi: https://doi. org/10.1590/S1806-37562015000000058 
23. Steffen TM, Hacker TA, Mollinger L. Age-and gender-related test performance in community-dwelling elderly people: Six-minute walk test, berg balance scale, timed up \& go test, and gait speeds. Physical Therapy. 2002;82(2):128137. doi: https://doi.org/10.1093/ptj/82.2.128

24. Hermes BM, Cardoso DM, Gomes TJN, Dos Santos TD, Vicente MS, Pereira SN, et al. Short-term inspiratory muscle training potentiates the benefits of aerobic and resistance training in patients undergoing CABG in phase II cardiac rehabilitation program. Rev Bras Cir Cardiovasc [internet]. 2015;30(4):474-81. doi: 10.5935 / 1678-9741.20150043

25. Janssens L, Brumagne S, McConnell AK, Claeys K, Pijnenburg M, Burtin C, et al. Proprioceptive changes impair balance control in individuals with chronic obstructive pulmonary disease. PLoS ONE. 2013;8(3):e57949. doi: https:// doi.org/10.1371/journal.pone.0057949

26. Robinovitch SN, Heller B, Lui A, Cortez J. Effect of strength and speed of torque development on balance recovery with the ankle strategy. J Neurophysiol. 2002;88: 613-620. doi: 10.1152 / jn.2002.88.2.613

27. Allum JH, Bloem BR, Carpenter MG, Hulliger M, Hadders-Algra M. Proprioceptive control of posture: a review of new concepts. Gait Posture. 1998;8(3):214-42. doi: 10.1016 / s0966-6362 (98) 00027-7

28. Chiappa GR, Roseguini BT, Vieira PJ, Alves CN, Tavares A, Winkelmann ER, et al. Inspiratory muscle training improves blood flow to resting and exercising limbs in patients with chronic heart failure. J Am Coll Cardiol [internet]. 2008;51(17):1663-71. doi: 10.1016 / j.jacc.2007.12.045

29. Pahor M, Guralnik JM, Ambrosius WT, Blair S, Bonds DE, Church TS, et al. Effect of structured physical activity on prevention of major mobility disability in older adults: The life study randomized clinical Trial. JAMA. 2014;311(23):23872396. doi: 10.1001 / jama.2014.5616

30. Miozzo AP, Stein C, Marcolino MZ, Sisto IR, Hauck M, Coronel CC, et al. Effects of high-intensity inspiratory muscle training associated with aerobic exercise in patients undergoing CABG: Randomized Clinical Trial. Braz J Cardiovasc Surg [internet]. 2018;33(4):376-83. doi: https://doi.org/10.21470/1678-9741-2018-0053 